\title{
Impact of high body mass index on allograft outcomes in kidney transplant recipients with presensitization to human leukocyte antigen
}

\author{
Yohan Park ${ }^{1,2}$, Hanbi Lee ${ }^{1,2}$, Eun Jeong Ko ${ }^{1,2}$, Sua Lee ${ }^{3}$, Tae Hyun Ban ${ }^{4}$, Ji-Won Min ${ }^{5}$, Hye-Eun Yoon ${ }^{6}$, \\ Eun-Jee $\mathrm{Oh}^{7}$, Chul Woo Yang ${ }^{1,2}$, Byung Ha Chung ${ }^{1,2}$ \\ ${ }^{1}$ Transplantation Research Center, Seoul St. Mary's Hospital, College of Medicine, The Catholic University of Korea, Seoul, Republic of Korea \\ ${ }^{2}$ Division of Nephrology, Department of Internal Medicine, Seoul St. Mary's Hospital, College of Medicine, The Catholic University of Korea, \\ Seoul, Republic of Korea \\ ${ }^{3}$ Division of Nephrology, Department of Internal Medicine, Seoul National University Hospital, College of Medicine, Seoul National University, \\ Seoul, Republic of Korea \\ ${ }^{4}$ Division of Nephrology, Department of Internal Medicine, Eunpyeong St. Mary's Hospital, College of Medicine, The Catholic University of \\ Korea, Seoul, Republic of Korea \\ ${ }^{5}$ Division of Nephrology, Department of Internal Medicine, Bucheon St. Mary's Hospital, College of Medicine, The Catholic University of Korea, \\ Seoul, Republic of Korea \\ ${ }^{6}$ Division of Nephrology, Department of Internal Medicine, Incheon St. Mary's Hospital, College of Medicine, The Catholic University of Korea, \\ Seoul, Republic of Korea \\ ${ }^{7}$ Department of Laboratory Medicine, Seoul St. Mary's Hospital, College of Medicine, The Catholic University of Korea, Seoul, Republic of Korea
}

Background: This study aimed to investigate whether high body mass index (BMI) and presensitization to human leukocyte antigen (HLA) in kidney transplant recipients (KTRs) affected allograft outcomes.

Methods: From January 2010 to December 2018, 1,290 kidney transplantations (KTs) were performed at the Seoul St Mary's Hospital. Of these, 682 cases of ABO-compatible living donor KT patients were enrolled. They were divided into four groups (low BMI-non-sensitized, high BMI-non-sensitized, low BMl-sensitized, and high BMI-sensitized) according to the median BMl value (22.7 $\mathrm{kg} / \mathrm{m}^{2}$ ) and HLA presensitization status (anti-HLA antibody mean fluorescence intensity $>3,000$ ). Short-term and long-term allograft outcomes were compared between groups.

Results: In the high BMI-sensitized group, the decline in allograft function was higher than that in the other three groups. Death-censored graft loss (DCGL) rates were highest in the high BMI-sensitized group (4 of 21 [19.0\%], p = 0.04). In the multivariable Cox regression hazard regression model analysis, the hazard ratio (HR) for DCGL was intensified when high BMI and presensitization statuses were combined (HR, 3.75; $p=0.03$ ); these statuses significantly interacted with each other ( $p$-value for interaction $=0.008$ ).

Received: November 12, 2020; Revised: December 5, 2020; Accepted: December 14, 2020

Editor: Woo Yeong Park, Keimyung University, Daegu, Republic of Korea

Correspondence: Byung Ha Chung

Division of Nephrology, Department of Internal Medicine, Seoul St. Mary's Hospital, College of Medicine, The Catholic University of Korea, 222

Banpo-daero, Seocho-gu, Seoul 06591, Republic of Korea. E-mail: chungbh@catholic.ac.kr

ORCID: https://orcid.org/0000-0003-0048-5717

Yohan Park's current affiliation is ‘Division of Nephrology, Department of Internal Medicine, Konyang University Hospital, College of Medicine, Konyang University, Daejeon, Republic of Korea.'

Copyright (C) 2021 by The Korean Society of Nephrology

(a) This is an Open Access article distributed under the terms of the Creative Commons Attribution Non-Commercial and No Derivatives License (http:// creativecommons.org/licenses/by-nc-nd/4.0/) which permits unrestricted non-commercial use, distribution of the material without any modifications, and reproduction in any medium, provided the original works properly cited. 
Conclusion: Our results suggest that presensitization to HLA and high BMI might have an interactive adverse impact on allograft outcomes in KTRs.

Keywords: Body mass index, Presensitization, Graft survival, Kidney transplantation

\section{Introduction}

Obesity is a major public health problem that is increasing worldwide [1], and its prevalence in Korea increased from $29.2 \%$ in 2001 to $34.6 \%$ in 2018 [2]. Obesity and metabolic syndromes are recognized as risk factors for the development and progression of chronic kidney disease (CKD) by various mechanisms [3,4]. In a previous nationwide cohort study in Korea, obesity (body mass index $[\mathrm{BMI}] \geq 25.0 \mathrm{~kg} / \mathrm{m}^{2}$ ) was identified as an independent risk factor for CKD progression [5]. Moreover, obesity also adversely affects allograft function in kidney transplant (KT) recipients (KTRs). A study reported that recipient obesity was an independent risk factor for death-censored graft loss (DCGL) and biopsy-proven acute rejection (BPAR) [6]. Another study also reported that recipient obesity was an independent risk factor for overall graft loss [7]. Furthermore, a meta-analysis reported that recipient obesity had a marginally greater risk for DCGL [8].

Pretransplant sensitization to human leukocyte antigen (HLA) is a well-known risk factor associated with adverse allograft outcomes [9]. Presensitization to HLA is not only associated with a high rate of acute antibody-mediated rejection (ABMR) but also with the gradual development of chronic allograft tissue injury caused by humoral immune system activation. Indeed, the development of chronic ABMR was significantly higher in patients with presensitization to HLA compared to patients with low immunologic risk [10-12]. Moreover, both preformed persistent donor-specific antibody (DSA) and preformed cleared DSA showed an increased risk of graft loss [9]. Another study reported that preformed donor-specific anti-HLA antibodies (HLA-DSAs) with mean fluorescence intensity (MFI) $>3,000$ had an increased risk of graft loss [13].

Therefore, both obesity and pretransplant sensitization to HLA in KTRs might contribute to the progression of chronic allograft tissue injury and adverse allograft outcomes, although the mechanisms are different. However, it has not been investigated whether both factors have an interactive effect on allograft outcomes. Hence, in this study, we analyzed the short- and long-term graft outcomes in KTRs with high BMI and presensitization to HLA and investigated the interaction between high BMI and HLA presensitization status.

\section{Methods}

\section{Study design}

This was a retrospective observational single-center study. Between January 2010 and December 2018, 1,290 KTs were performed at the Seoul St. Mary's Hospital in Seoul, South Korea. Of these, 412 patients received a kidney from a deceased donor, 195 cases were ABO-incompatible KTs, and 1 KTR had both legs amputated; these cases were excluded from the study. Finally, 682 KTRs were included in this analysis. The distribution of BMI in the KTRs is presented in Supplementary Fig. 1 (available online), and the median BMI value was $22.7 \mathrm{~kg} / \mathrm{m}^{2}$. Patients with BMI of $\geq 22.7 \mathrm{~kg} / \mathrm{m}^{2}$ were categorized in the high BMI group, while others were classified in the low BMI group. The cases were defined as presensitized to HLA when the MFI value of HLA-DSA at baseline was higher than 3,000 [13] and as non-sensitized when the value was below 3,000. Based on the above classifications, KTRs were divided into four groups: low BMI-non-sensitized, high BMI-non-sensitized, low BMI-sensitized, and high BMI-sensitized as presented in Fig. 1. This study followed the Declaration of Helsinki and was approved by the Institutional Review Board (IRB) of Seoul St. Mary's Hospital (No. XC15RIMI0072K). As it was a retrospective study using data obtained from medical records, informed consent was waived by the IRB.

\section{Human leukocyte antigen typing, human leukocyte antigen antibodies, and donor-specific antibody}

HLA typing and HLA antibodies were measured as described previously $[14,15]$. Briefly, HLA-A, HLA-B, HLA-DR, and HLA-DQB1 typing was performed using deoxyribonucleic acid molecular typing with sequence-specific oligonucle- 


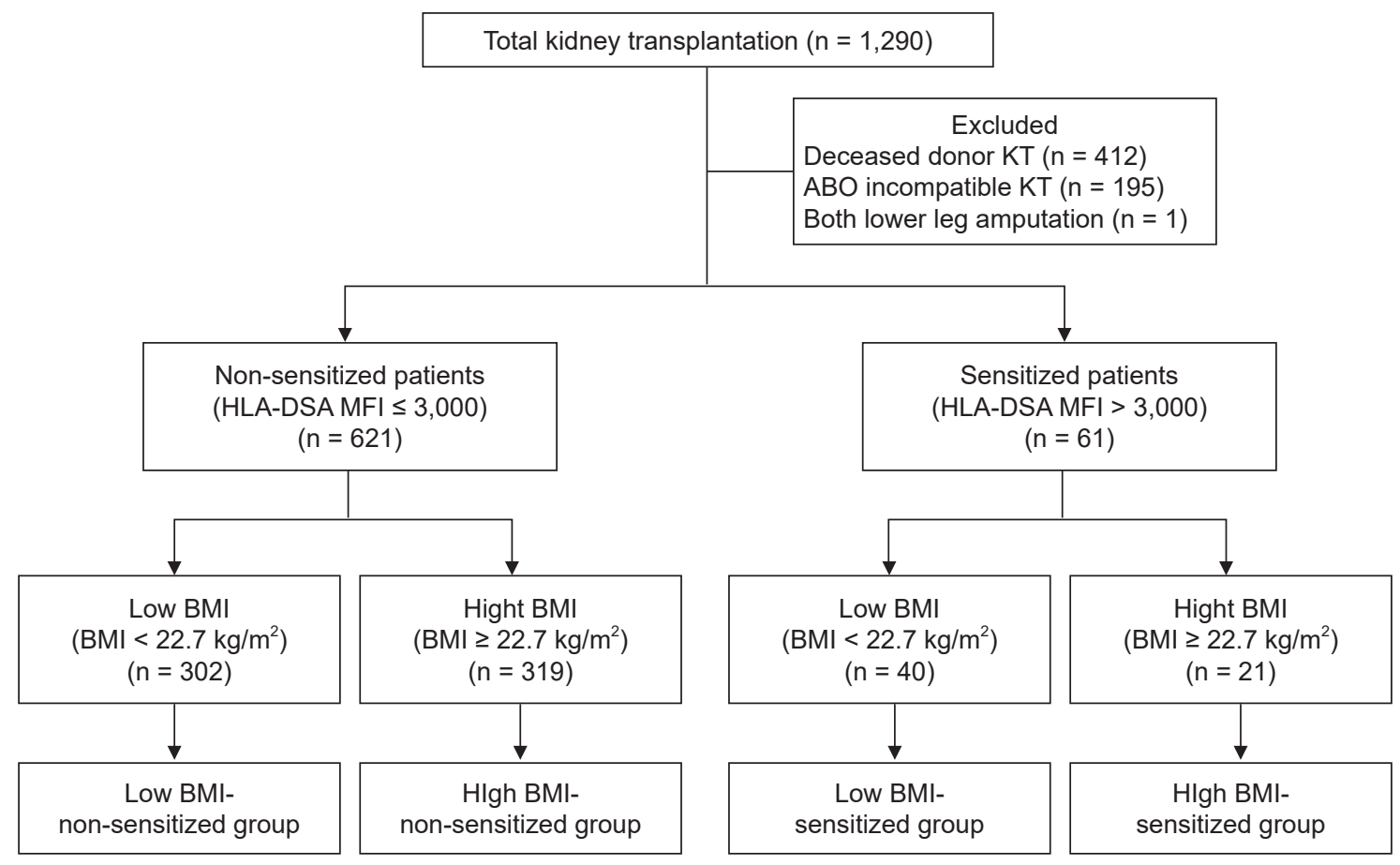

Figure 1. Distribution of the patient population according to BMI and HLA presensitization status. Of the 682 kidney transplant recipients of ABO-compatible living donor kidney transplantations (KTs), 621 cases were HLA-non-sensitized, while 61 cases were HLAsensitized. Patients were categorized into four groups according to the median BMI: low BMI-non-sensitized $(n=302)$, high BMI-nonsensitized ( $n=319)$, low BMI-sensitized $(n=40)$, and high BMI-sensitized $(n=21)$ groups.

BMI, body mass index; HLA, human leukocyte antigen; HLA-DSA, donor-specific anti-human leukocyte antigen antibody; MFI, mean fluorescence intensity.

otide probes with Lifecodes HLA SSO typing kits (Immucor, Stamford, CT, USA). Lifecodes LSA Class I and Class II kits (Gen-Probe Transplant Diagnostic Inc., Stamford, CT, USA) or LABScreen Single Antigen (One Lambda Inc., Thermo Fisher Scientific, Canoga Park, CA, USA) were used to detect HLA antibodies in the recipient sera. The manufacturer's instructions were followed and $10 \mu \mathrm{L}$ of each serum sample was used. The fluorescence intensities of the samples were measured using a Luminex 200 system (Luminex Corp., Austin, TX, USA).

\section{Desensitization protocols for presensitized patients}

The desensitization protocol in our center has been described previously [16-18]. Briefly, the desensitization protocol for HLA presensitized patients consisted of rituximab, total plasma exchange (TPE), and intravenous immunoglobulin (IVIG). Rituximab was administered 2 weeks to 1 month before the transplantation and TPE was performed seven times using $5 \%$ albumin and fresh frozen plasma. The control of TPE frequency was based on the MFI titer of HLADSAs. IVIG was administered at a dose of $100 \mathrm{mg} / \mathrm{kg}$ for 1 hour after every TPE. In patients with an HLA-DSA MFI titer of 1,000 to 3,000 or panel reactive antibody (PRA, Class I or Class II) of $>50 \%$ with no HLA-DSAs, only rituximab was administered before transplantation. In all patients who underwent desensitization, prophylactic agents were used to prevent Pneumocystis jirovecii pneumonia (PJP) and cytomegalovirus (CMV) infection. If the crossmatch (XM) test of T-cell complement-dependent cytotoxicity (CDC) was positive or HLA-DSAs were present, and the MFI of HLA-DSAs did not decrease adequately after three cycles of TPE, a bortezomib-based protocol was used, in which bortezomib was administered four times in addition to the desensitization protocol.

\section{Clinical parameters and outcomes}

The age, sex, height, and weight of the donor and estimated glomerular filtration rate (eGFR) based on the Chronic 
Kidney Disease-Epidemiology Collaboration (CKD-EPI) equation were collected as baseline characteristics. The age, height, and weight of the KTR and the Mosteller body surface area (BSA) ratio of donor to recipient, history of diabetes mellitus (DM) and hypertension (HTN), cause of end-stage renal disease (ESRD), previous dialysis modality, previous dialysis period, and previous KT history were collected as baseline demographic characteristics. Total cholesterol, triglyceride, high-density lipoprotein (HDL)-cholesterol, low-density lipoprotein (LDL)-cholesterol, hemoglobin Alc levels, and hepatitis $\mathrm{C}$ virus (HCV) seropositivity rates were obtained from pretransplant investigations. The results of the XM test using CDC and flow cytometry crossmatch (FCXM), HLA-DSA and MFI results by Luminex single antigen assay, and PRA titers were obtained as a pretransplant immunoassay. Transplantation information included mismatch number, type of induction therapy, the main immunosuppressant used, and drugs used for desensitization.

We analyzed the incidence of BPAR within 1-year of transplantation (early acute rejection), CMV infection, BK viremia, and PJP rates as short-term clinical outcomes in the four groups. The variables used for analyzing long-term clinical outcomes included BPAR incidence after 1-year of transplantation (late acute rejection), chronic active ABMR, and biopsy-proven calcineurin inhibitor (CNI) toxicity rates. DCGL and patient death rates were also analyzed.

CMV infection and BK viremia were screened with CMV real-time quantitative (RQ) polymerase chain reaction (PCR) and BK virus real-time (RT) PCR through blood tests at 1- to 2 -month intervals until 1 year after transplantation. From 1 year after transplantation, screening was performed with CMV RQ-PCR and BKV RT-PCR every 6 months to 1 year. Moreover, CMV RQ-PCR and BKV RT-PCR tests were performed when renal function deterioration occurred or when the clinician determined that the tests were necessary.

Allograft kidney biopsy was performed in cases of unexpected renal allograft dysfunction (serum creatinine of $25 \%$ above the baseline), unexpected development of proteinuria, and development of de novo HLA-DSA. Allograft kidney biopsy findings were interpreted according to the Banff classification in 2009. BPAR was diagnosed with allograft biopsy as suitable for acute T-cell mediated rejection (TCMR) and acute ABMR criteria according to the Banff classification. Similarly, chronic active ABMR and biopsy-proven CNI toxicity were diagnosed with allograft biopsies according to the Banff clas- sification [19]. Death-censored allograft survival duration was defined as the period from KT to dialysis or preemptive KT, except for patient death in a functioning allograft. Patient survival duration was defined as the period from KT to death due to any cause. The data of changes in allograft function based on serum creatinine levels were collected until 4 years after KT.

The primary outcome of this study was to compare the impact of BMI on DCGL in non-sensitized and sensitized patients. Secondary outcomes of this study were early acute rejection, CMV infection, BK viremia, PJP, late acute rejection, chronic active ABMR, biopsy-proven CNI toxicity, patient death rates, and eGFR based on the CKD-EPI equation [20].

\section{Statistical analysis}

All continuous variables were expressed as mean \pm standard deviation. If the variables followed a normal distribution, an analysis of variance was performed. If the variables showed a non-normal distribution, a Kruskal-Wallis test was performed. Tukey or Dunnett T3 method was performed for post hoc analysis. All categorical variables were compared using the chi-square test or Fisher exact test and expressed as proportions. A multivariable Cox hazard regression model analysis was performed to determine the risk factors affecting DCGL and to investigate the interaction between high BMI and HLA presensitivity. Cumulative survival rates were analyzed during the follow-up period in the four groups by Kaplan-Meier survival analysis. Causes of DCGL and patient death were compared using the chi-square test or Fisher exact test. The mean eGFR (CKD-EPI) and standard deviation in the four groups were evaluated. All statistical analyses were performed using IBM SPSS version 24 (IBM Corp., Armonk, NY, USA) and Microsoft Excel 2016 (Microsoft, Redmond, WA, USA).

\section{Results}

Comparison of baseline clinical and laboratory parameters according to body mass index and human leukocyte antigen presensitization status

The mean duration of follow-up of the patients included in this study was 61.8 months. The baseline characteristics of the four groups are shown in Table 1, 2. Among donor fac- 
Table 1. Comparison of baseline characteristics of donor factors among the four groups according to BMI and presensitization status

\begin{tabular}{|c|c|c|c|c|c|}
\hline \multirow{2}{*}{ Characteristic } & \multicolumn{4}{|c|}{ Group } & \multirow{2}{*}{$p$-value } \\
\hline & Low BMI-non-sensitized & High BMI-non-sensitized & Low BMI-sensitized & High BMI-sensitized & \\
\hline No. of patients & 302 & 319 & 40 & 21 & \\
\hline Age, $\geq 65$ yr & $2(0.7)$ & $7(2.2)$ & $0(0)$ & $1(4.8)$ & 0.20 \\
\hline Male sex & $146(48.3)$ & $129(40.4)$ & $20(50.0)$ & $13(61.9)$ & 0.08 \\
\hline \multicolumn{6}{|l|}{$\mathrm{BMI}\left(\mathrm{kg} / \mathrm{m}^{2}\right)$} \\
\hline < 18.5 (underweight) & $12(4.0)$ & $8(2.5)$ & $0(0)$ & $1(4.8)$ & 0.45 \\
\hline 18.5-23.0 (normal) & 121 (40.1) & $113(35.4)^{a}$ & $21(52.5)^{b, c}$ & $4(19.0)^{a}$ & 0.04 \\
\hline 23.0-25.0 (overweight) & $75(24.8)$ & $88(27.6)$ & $6(15.0)$ & $8(38.1)$ & 0.19 \\
\hline 25.0-30.0 (mildly obese) & $78(25.8)$ & $95(29.8)$ & $12(30.0)$ & $5(23.8)$ & 0.69 \\
\hline$\geq 30.0$ (grossly obese) & $16(5.3)$ & $15(4.7)$ & $1(2.5)$ & $3(14.3)$ & 0.23 \\
\hline eGFR $\left(\mathrm{mL} / \mathrm{min} / 1.73 \mathrm{~m}^{2}\right)$ & $114.3 \pm 12.6$ & $113.5 \pm 13.1$ & $117.3 \pm 11.7$ & $113.1 \pm 10.5$ & 0.33 \\
\hline
\end{tabular}

Data are expressed as number only, number (\%), or mean \pm standard deviation.

$\mathrm{BMI}$, body mass index; eGFR, estimated glomerular filtration rate.

${ }^{a} \mathrm{p}<0.05$ vs. low BMI-sensitized group, ${ }^{b} p<0.05$ vs. high BMI-non-sensitized group, and ${ }^{c} p<0.05$ vs. high BMl-sensitized group.

tors, there were no statistical differences among the groups in terms of old age, sex, and eGFR; however, the normal weight of the donors (BMI, $18.5-23.0 \mathrm{~kg} / \mathrm{m}^{2}$ ) was relatively higher in the low BMI-sensitized group (21 of 40, 52.5\%; p $=0.04$ ) than those in the other groups. Regarding the clinical demographics of recipients, the proportion of elderly patients was not significantly different among the four groups, but the proportion of male patients was higher in the non-sensitized groups, especially in the high BMI-non-sensitized group ( 235 of $319,73.7 \%$; $p<0.001$ ). According to BMI categories, grossly obese patients were observed only in the high BMI-non-sensitized group (25 of 319, 7.8\%; $\mathrm{p}<0.001$ ). The donor/recipient (D/R) BSA ratio was significantly lower in the high BMI-non-sensitized group than in other groups $(0.94 \pm 0.13, p<0.001)$. The proportions of recipients with DM and HTN were higher in the high BMI groups $(\mathrm{p}<0.001$, for each). The mean triglyceride and hemoglobin Alc levels were significantly higher, and HDL- cholesterol levels were lower in both high BMI groups ( $\mathrm{p}<0.001$, for each). Regarding factors that cause ESRD, previous renal diseases had high proportions of DM in the high BMI groups, and other causes showed various distributions between groups. Among the types of dialysis modality, the proportion of peritoneal dialysis was the highest in the high BMI-non-sensitized group (47 of $319,14.7 \%$; $\mathrm{p}=0.02$ ).

In the baseline immunologic test, the proportions with a positive XM test through both CDC and FCXM were significantly higher in the sensitized groups ( $p<0.001$ for each) than in the non-sensitized groups; however, no difference in the rate of positivity was seen within the sensitized groups according to BMI status. The MFI values of HLA-DSAs were significantly higher in the sensitized groups than in the non-sensitized groups. The proportion of previous KT history was the highest in the low BMI-sensitized group (13 of 40, 32.5\%; $\mathrm{p}<0.001$ ). Antithymocyte globulin was used as induction therapy, and the proportion of desensitization therapy was significantly higher in the sensitized groups $(\mathrm{p}<$ 0.001 for each).

\section{Comparison of short- and long-term clinical outcomes and allograft functions according to body mass index and presensitization status}

Table 3 shows the short- and long-term clinical outcomes of the compared groups. In the short-term clinical outcomes, the rates of early ABMR were significantly higher in the sensitized groups $(\mathrm{p}<0.001)$, but there was no significant difference within the sensitized groups according to BMI status. CMV infection and BK viremia rates tended to be higher in the sensitized groups but without statistical significance ( $p$ $=0.08$ and $\mathrm{p}=0.18$, respectively). The median duration until CMV infection after transplantation was 33 days, and the median duration until BK viremia was 99 days. Early TCMR and PJP rates did not show significant differences between the four groups. In long-term clinical outcomes, the rate of late ABMR tended to be the highest in the high BMI-sensitized group, although the difference was not significant (2 of $21,9.5 \%$; $=0.15$ ). There were no significant differences 
Table 2. Comparison of baseline characteristics of recipient factors among the four groups according to BMI and presensitization status

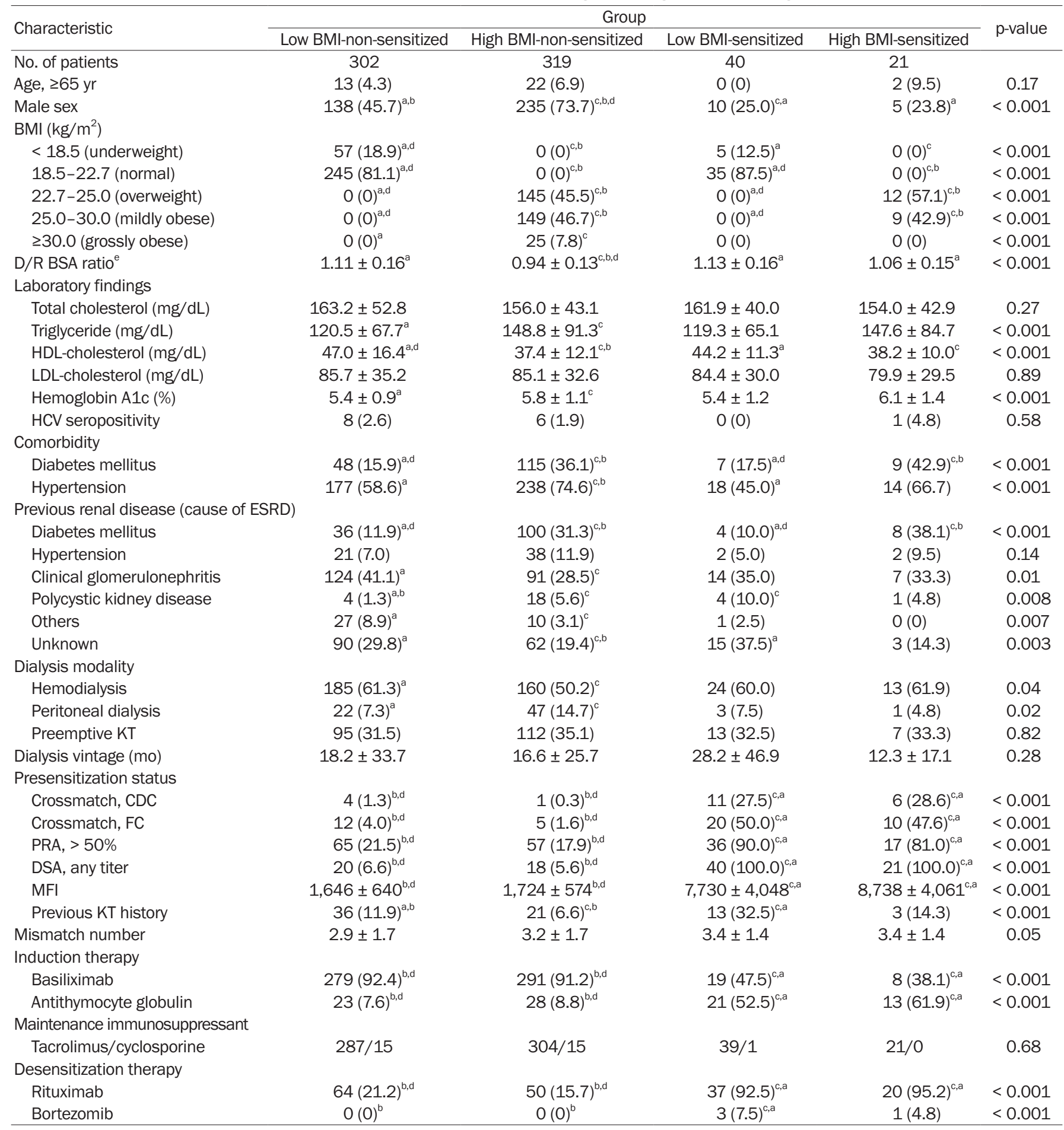

Data are expressed as number only, number (\%), or mean \pm standard deviation.

$\mathrm{BMI}$, body mass index; BSA, body surface area; CDC, complement-dependent cytotoxicity; D/R, donor/recipient; DSA, donor-specific antibody; ESRD, endstage renal disease; FC, flow cytometry; HCV, hepatitis C virus; HDL, high-density lipoprotein; KT, kidney transplantation; LDL, low-density lipoprotein; MFI, mean fluorescence intensity; PRA, panel reactive antibody.

${ }^{a} p<0.05$ vs. high BMI-non-sensitized group, ${ }^{b} p<0.05$ vs. low BMI-sensitized group, ${ }^{c} p<0.05$ vs. low BMI-non-sensitized group, ${ }^{d} p<0.05$ vs. high BMIsensitized group. ${ }^{e} \mathrm{BSA}$ was calculated using the Mosteller body surface area equation. 
Table 3. Comparison of short- and long-term clinical outcomes among the four groups according to BMI and presensitization status

\begin{tabular}{|c|c|c|c|c|c|}
\hline \multirow[b]{2}{*}{ Outcome } & \multicolumn{4}{|c|}{ Group } & \multirow[b]{2}{*}{ p-value } \\
\hline & $\begin{array}{l}\text { Low BMI-non-sensitized } \\
\qquad(\mathrm{n}=302)\end{array}$ & $\begin{array}{l}\text { High BMI-non-sensitized } \\
\qquad(\mathrm{n}=319)\end{array}$ & $\begin{array}{l}\text { Low BMl-sensitized } \\
\qquad(\mathrm{n}=40)\end{array}$ & $\begin{array}{l}\text { High BMI-sensitized } \\
(\mathrm{n}=21)\end{array}$ & \\
\hline \multicolumn{6}{|l|}{ Short-term outcome } \\
\hline Early $\mathrm{ABMR}^{\mathrm{a}}$ & $13(4.3)^{b, c}$ & $12(3.8)^{b, c}$ & $16(40.0)^{\mathrm{d}, \mathrm{e}}$ & $6(28.6)^{d, e}$ & $<0.001$ \\
\hline Early TCMR ${ }^{\mathrm{a}}$ & $32(10.6)$ & $47(14.7)$ & $4(10.0)$ & $1(4.8)$ & 0.28 \\
\hline CMV infection & $34(11.3)$ & $33(10.3)$ & $6(15.0)$ & $6(28.6)$ & 0.08 \\
\hline BK viremia & $44(14.6)$ & $39(12.2)$ & $9(22.5)$ & $5(23.8)$ & 0.18 \\
\hline PJP & $3(1.0)$ & $7(2.2)$ & $1(2.5)$ & $1(4.8)$ & 0.46 \\
\hline \multicolumn{6}{|l|}{ Long-term outcome } \\
\hline Late $\mathrm{ABMR}^{f}$ & $6(2.0)$ & $8(2.5)$ & $2(5.0)$ & $2(9.5)$ & 0.15 \\
\hline Late $\mathrm{TCMR}^{f}$ & $13(4.3)$ & $9(2.8)$ & $2(5.0)$ & $1(4.8)$ & 0.74 \\
\hline Chronic active ABMR & $4(1.3)$ & $13(4.1)$ & $1(2.5)$ & $1(4.8)$ & 0.20 \\
\hline Biopsy-proven CNI toxicity & $18(6.0)$ & $23(7.2)$ & $1(2.5)$ & $1(4.8)$ & 0.67 \\
\hline
\end{tabular}

Data are expressed as number (\%).

BMI, body mass index; ABMR, acute antibody-mediated rejection; BPAR, biopsy-proven acute rejection; CMV, cytomegalovirus; CNI, calcineurin inhibitor; TCMR, T-cell mediated rejection; PJP, Pneumocystis jiroveci pneumonia.

${ }^{a}$ Early acute rejection (ABMR, TCMR) is the occurrence of BPAR within 1-year of transplantation. ${ }^{b} p<0.05$ vs. low BMI-sensitized group, ${ }^{c} p<0.05$ vs. high BMI-sensitized group, ${ }^{d} p<0.05$ vs. low BMI-non-sensitized group, ${ }^{e} p<0.05$ vs. high BMI-non-sensitized group. ${ }^{f}$ Late acute rejection (ABMR, TCMR) is the occurrence of BPAR after 1-year of transplantation.

in the rates of late TCMR, chronic active ABMR, and biopsy-proven $\mathrm{CNI}$ toxicity between the groups.

Fig. 2 shows the change in mean eGFR during the follow-up period after transplantation in the four groups. Although eGFR did not show significant differences between the compared groups, the decline in eGFR was higher in the high BMI-sensitized group than in the other three groups. Moreover, in this group, eGFR was less than $60 \mathrm{~mL} / \mathrm{min} / 1.73$ $\mathrm{m}^{2}$ after 3 years of KT, while it was more than $60 \mathrm{~mL} / \mathrm{min} / 1.73$ $\mathrm{m}^{2}$ in the other groups.

\section{Comparison of death-censored graft loss rate and its causes according to body mass index and presensitization status}

In all, 44 DCGL events occurred; of these, 14 were in the low BMI-non-sensitized group (14 of 302, 4.6\%), 22 in the high BMI-non-sensitized group (22/319, 6.9\%), and four in the low BMI-sensitized $(4 / 40,10.0 \%)$ and high BMI-sensitized groups $(4 / 21,19.0 \%)$. respectively (Table 4$)$. There were no statistically significant differences in the causes of DCGL between the four groups. In the Kaplan-Meier survival analysis, the graft survival rate decreased significantly in the sensitized groups, and it was the poorest in the high BMI-sensitized group (Fig. 3, log-rank $p=0.007$ ).

Table 5 shows the results of the multivariable Cox hazard regression model analysis. Model 1 adjusted baseline char- acteristics showed significant differences among the four groups, except for dialysis modality and vintage, and the variables were as follows: donor factors (age $\geq 65$ years, sex, BMI categories, and eGFR), recipient factors (age $\geq 65$ years, sex, D/R BSA ratio, triglyceride, HDL-cholesterol, hemoglobin Alc, HCV seropositivity, comorbidities, previous renal disease, previous KT history, PRA $>50 \%$, and mismatch number). Model 2 included dialysis modality and vintage as adjusted variables. High BMI alone did not show a significant hazard ratio (HR) in any of the DCGL models. Presensitization status was observed as an independent risk factor for DCGL (univariable $\mathrm{HR}=2.73, \mathrm{p}=0.01$, adjusted $\mathrm{HR}=2.90, \mathrm{p}=0.007$ in model 1 ; and adjusted HR $=3.63, \mathrm{p}=0.004$ in model 2). Interestingly, when high BMI and presensitization status were combined, HR increased further and was identified as a significant independent risk factor (univariable $\mathrm{HR}=$ 4.06, $\mathrm{p}=0.008$, adjusted HR $=3.82, \mathrm{p}=0.01$ in model 1 ; and adjusted HR = 3.75, $\mathrm{p}=0.03$ in model 2). Moreover, high BMI and presensitization status showed a statistically significant interaction for DCGL ( $p$-value for interaction $=0.008$ ).

\section{Comparison of patient death rate and its causes according to body mass index and presensitization status}

Overall, 21 patients died; however, there was no significant difference in the rate of death between the four groups; 8 


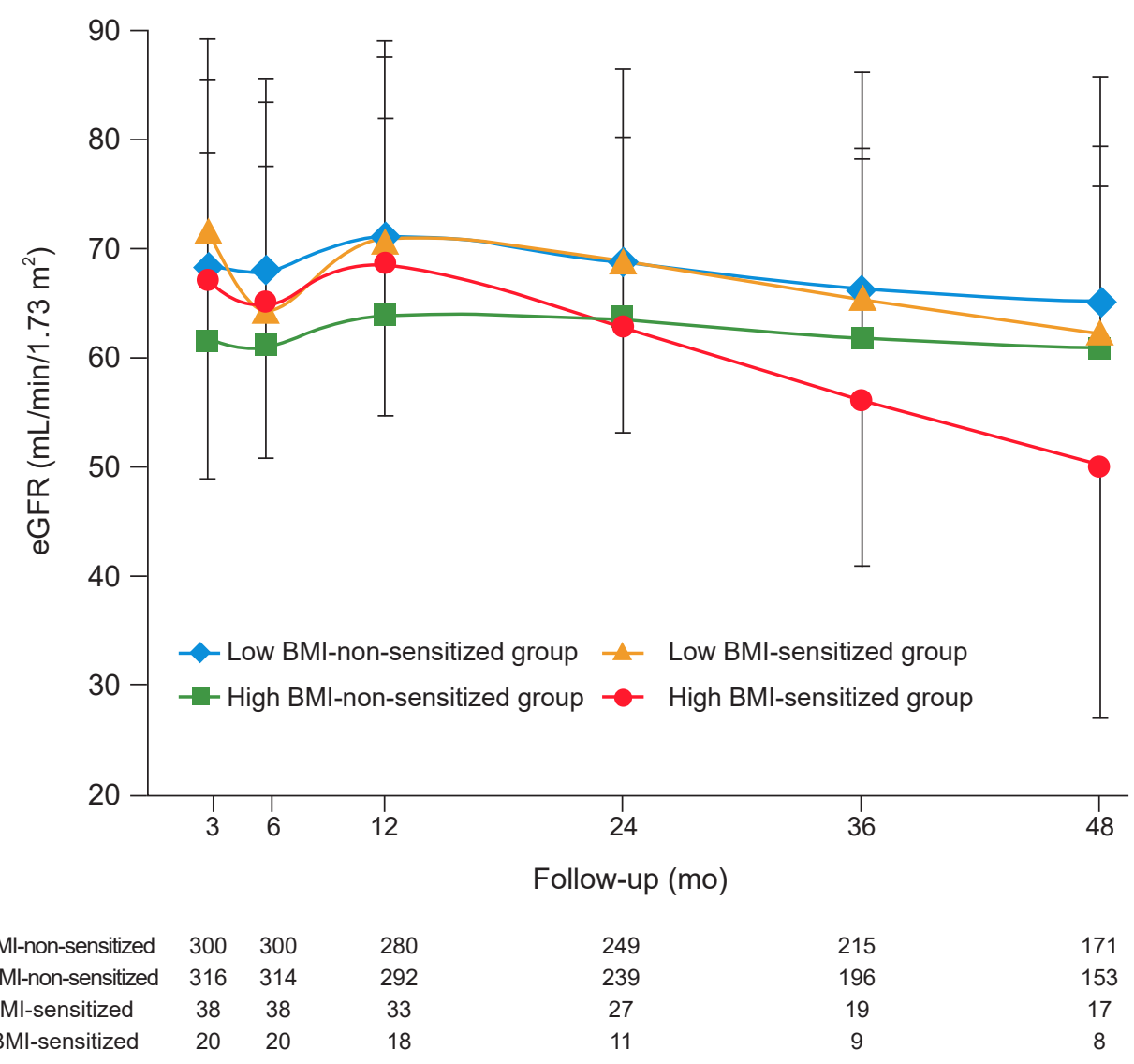

Figure 2. The eGFR during the follow-up period in the four groups according to BMI and HLA presensitization status. Note that the decrease in allograft function tended to be higher in the high BMl-sensitized group.

$\mathrm{BMI}$, body mass index; eGFR, estimated glomerular filtration rate; HLA, human leukocyte antigen.

Table 4. Comparison of death-censored graft loss, overall graft loss, and patient death rates among the four groups according to BMI and presensitization status

\begin{tabular}{|c|c|c|c|c|c|}
\hline \multirow[b]{2}{*}{ Variable } & \multicolumn{4}{|c|}{ Group } & \multirow[b]{2}{*}{ p-value } \\
\hline & $\begin{array}{l}\text { Low BMI-non-sensitized } \\
\qquad(\mathrm{n}=302)\end{array}$ & $\begin{array}{l}\text { High BMI-non-sensitized } \\
\qquad(\mathrm{n}=319)\end{array}$ & $\begin{array}{l}\text { Low BMl-sensitized } \\
\qquad(n=40)\end{array}$ & $\begin{array}{l}\text { High BMI-sensitized } \\
\qquad(n=21)\end{array}$ & \\
\hline Death-censored graft loss & $14(4.6)^{a}$ & $22(6.9)$ & $4(10.0)$ & $4(19.0)^{b}$ & 0.04 \\
\hline Overall graft loss & $20(6.6)$ & $30(9.4)$ & $5(12.5)$ & $4(19.0)$ & 0.14 \\
\hline Patient death & $8(2.6)$ & $12(3.8)$ & $1(2.5)$ & $0(0)$ & 0.71 \\
\hline
\end{tabular}

Data are expressed as number (\%).

BMI, body mass index.

${ }^{a} p<0.05$ vs. high BMl-sensitized group, ${ }^{b} p<0.05$ vs. low BMI-non-sensitized group.

deaths occurred in the low BMI-non-sensitized group (8 of $302,2.6 \%$ ), 12 in the high BMI-non-sensitized group (12 of $319,3.8 \%$ ), 1 in the low BMI-sensitized group (1 of 40, 2.5\%), and none in the high BMI-sensitized group (Table 4). When comparing the causes of death between the groups, there were no significant differences (Table 6).

\section{Discussion}

In this study, we analyzed the short- and long-term allograft outcomes according to the high BMI and HLA presensitization status of the recipients. We found that the high BMI-sensitized group had the most significant decline in allograft function among the four groups and the highest DCGL rates. Moreover, 


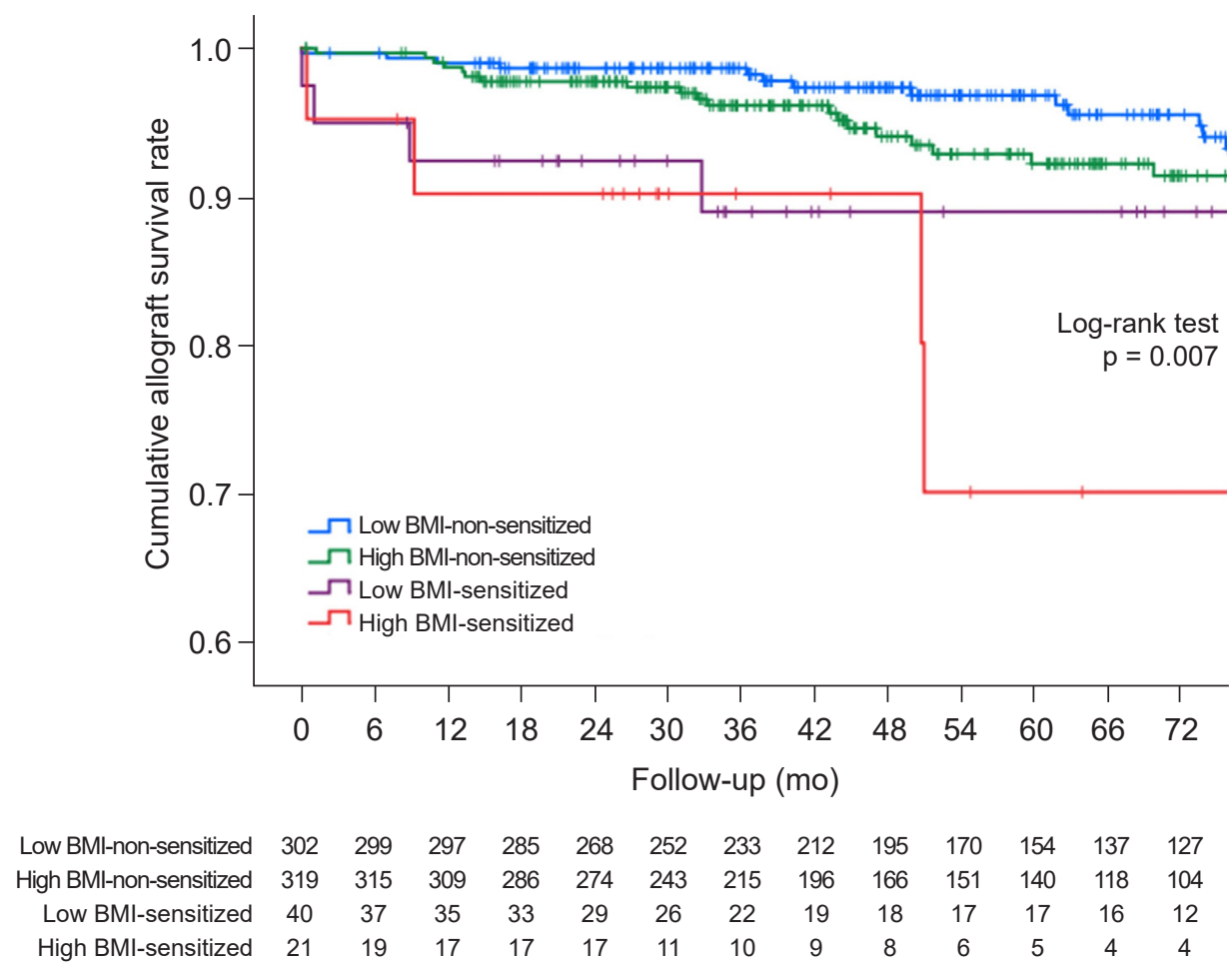

Figure 3. Kaplan-Meier survival analysis among the four groups according to BMI and human leukocyte antigen presensitization status for death-censored graft loss. Cumulative graft survival rates were significantly lower in the high BMI-sensitized group than in the low BMI-non-sensitized group (log-rank $p=0.001$ ) and high BMI-non-sensitized group (log-rank $p=0.02$ ) in post hoc analyses.

BMI, body mass index.

Table 5. Cox proportional hazard ratio (HR) model analysis for death-censored graft loss

\begin{tabular}{|c|c|c|c|c|c|c|}
\hline Variable & $\begin{array}{l}\text { Univariable HR (95\% } \\
\mathrm{Cl})\end{array}$ & $\begin{array}{l}\text { p-value for } \\
\text { interaction }\end{array}$ & $\begin{array}{c}\text { Model } 1^{\mathrm{a}}, \\
\text { multivariable HR } \\
(95 \% \mathrm{Cl})\end{array}$ & $\begin{array}{l}\text { p-value for } \\
\text { interaction }\end{array}$ & $\begin{array}{c}\text { Model } 2^{\mathrm{b}}, \\
\text { multivariable HR } \\
(95 \% \mathrm{Cl})\end{array}$ & $\begin{array}{l}p \text {-value for } \\
\text { interaction }\end{array}$ \\
\hline Low BMI & Reference & - & - & - & - & - \\
\hline High BMI & $1.60(0.88-2.93)$ & - & - & - & - & - \\
\hline Non-presensitization & Reference & - & - & - & - & \\
\hline Presensitization & $2.73(1.27-5.88)$ & - & - & - & - & - \\
\hline High BMI \& presensitization & $4.06(1.45-11.36)$ & 0.008 & $3.82(1.36-10.73)$ & 0.01 & $3.75(1.12-12.62)$ & 0.03 \\
\hline Mismatch number & $1.28(1.07-1.53)$ & - & $1.28(1.06-1.53)$ & - & $1.34(1.08-1.65)$ & - \\
\hline Hemodialysis & $0.90(0.50-1.64)$ & - & - & - & $0.39(0.19-0.81)$ & - \\
\hline
\end{tabular}

$\mathrm{BMI}$, body mass index; $\mathrm{Cl}$, confidence interval.

${ }^{a}$ Model 1 was adjusted with parameters showing significant differences among the four groups according to BMl and presensitization status. Excluding recipients with missing values, 673 (98.7\%) were included in Model 1. The following parameters were used: donor factors (age $\geq 65$ years, sex, BMI categories, estimated glomerular filtration rate), recipient factors (age $\geq 65$ years, sex, donor/recipient body surface area ratio, triglyceride, high-density lipoprotein-cholesterol, hemoglobin A1c, hepatitis C virus seropositivity, comorbidities, previous renal disease, previous kidney transplantation history, panel reactive antibody $>50 \%$, and mismatch number). ${ }^{b}$ Model 2 was adjusted with dialysis modality and dialysis vintage in addition to the variables included in Model 1. Excluding recipients with missing values, 448 (65.7\%) were included in Model 2.

a high BMI with presensitization status was found to be an independent risk factor for DCGL. There was also a significant interaction between high BMI and pretransplant HLA sensitivity leading to adverse allograft outcomes.
In a previous study that analyzed BMI and allograft outcomes for 51,927 recipients from 1988 to 1997 in the United States Renal Database, the relative risk of graft loss (1.07, $\mathrm{p}=$ 0.047 ) increased significantly when BMI $\geq 28.0 \mathrm{~kg} / \mathrm{m}^{2}$. The 
Table 6. Comparison of the causes of graft loss and patient death among the four groups according to BMI and presensitization status

\begin{tabular}{|c|c|c|c|c|c|}
\hline Variable & Low BMI-non-sensitized & High BMI-non-sensitized & Low BMI-sensitized & High BMI-sensitized & p-value \\
\hline Cause of graft loss & 14 & 22 & 4 & 4 & \\
\hline Acute rejection & $2(14.3)$ & $7(31.8)$ & $2(50.0)$ & $2(50.0)$ & 0.33 \\
\hline Chronic active TCMR/ABMR & $2(14.3)$ & $4(18.2)$ & $0(0)$ & $1(25.0)$ & 0.77 \\
\hline BKVAN & $1(7.1)$ & $1(4.5)$ & $0(0)$ & $1(25.0)$ & 0.47 \\
\hline Recurrent glomerulonephritis & $3(21.4)$ & $1(4.5)$ & $0(0)$ & $0(0)$ & 0.27 \\
\hline Others & $2(14.3)^{a}$ & $4(18.2)^{b}$ & $2(50.0)^{c}$ & $0(0)$ & 0.29 \\
\hline Unknown & $4(28.6)$ & $5(22.7)$ & $0(0)$ & $0(0)$ & 0.44 \\
\hline Cause of patient death & 8 & 12 & 1 & 0 & \\
\hline Cardiac & $1(12.5)$ & $2(16.7)$ & $0(0)$ & $0(0)$ & 0.89 \\
\hline Pulmonary & $0(0)$ & $1(8.3)$ & $0(0)$ & $0(0)$ & 0.68 \\
\hline Vascular & $0(0)$ & $2(16.7)$ & $0(0)$ & $0(0)$ & 0.44 \\
\hline Infection & $4(50.0)$ & $3(25.0)$ & $1(100)$ & $0(0)$ & 0.23 \\
\hline Malignancy & $3(37.5)$ & $3(25.0)$ & $0(0)$ & $0(0)$ & 0.68 \\
\hline Unknown & $0(0)$ & $1(8.3)$ & $0(0)$ & $0(0)$ & 0.68 \\
\hline
\end{tabular}

Data are expressed as number only or number (\%).

ABMR, antibody-mediated rejection; BKVAN, BK virus-associated nephropathy; BMI, body mass index; TCMR, T-cell mediated rejection.

${ }^{a}$ Graft loss causes of others were postoperative bleeding and amyloidosis. ${ }^{b}$ Graft loss causes of others were drug-induced nephropathy, sepsis, oxalate nephropathy, and thrombotic microangiopathy. ${ }^{\circ}$ Graft loss causes of others were two cases of postoperative bleeding.

authors suggested the effects of various comorbidities and proinflammatory cytokines expressed in obesity were a reason for the poor allograft outcomes in obesity [21]. Additionally, a recent study reported that the risk of acute rejection may increase due to the relative underdosing of immunosuppressants in obese recipients [22]. However, in studies after 2000, obesity was not identified as a significant risk factor for graft loss (risk ratio, 0.99; 95\% confidence interval, 0.83-1.19) [23]. Moreover, in a study that directly compared recipients from 1987 to 1999 and recipients from 2000 to 2016 in the Organ Procurement and Transplantation Network/United Network for Organ Sharing database, high BMI $\left(\geq 30.0 \mathrm{~kg} / \mathrm{m}^{2}\right)$ was still an independent risk factor for graft loss, but the relative risk significantly decreased after 2000 [24]. The authors suggested that the recent development of an immunosuppressive regimen and general medical practice for comorbidities in transplant recipients may be the reason for this phenomenon $[23,24]$.

The International Obesity Task Force of the World Health Organization (WHO) recommends that the Asian population sets the BMI value of obesity to $25.0 \mathrm{~kg} / \mathrm{m}^{2}$ and the BMI value of overweight to $23.0 \mathrm{~kg} / \mathrm{m}^{2}$ when analyzing comorbidity risk [25]. Previous studies that reported a worse allograft outcome in obesity (BMI $\geq 30.0 \mathrm{~kg} / \mathrm{m}^{2}$ ) were not studies involving Asian populations $[7,21,23,24]$. We considered that setting the obesity criterion to BMI of $25.0 \mathrm{~kg} / \mathrm{m}^{2}$ according to the WHO recommendation would be appropriate for the Asian population in this study. Similar results were obtained when patients in this study were classified by using obesity (BMI $\left.\geq 25.0 \mathrm{~kg} / \mathrm{m}^{2}\right)$, and the DCGL rate in the obesity-sensitized group was higher than that in the high BMI-sensitized group classified by BMI of $\geq 22.7 \mathrm{~kg} / \mathrm{m}^{2}$ (Supplementary Table 1, available online). Furthermore, the median BMI value of $22.7 \mathrm{~kg} / \mathrm{m}^{2}$ in this study, was close to the Asian population overweight cutoff value, and most patients in the high BMI group met the Asian overweight criteria.

In a recent study of 296,807 adult recipients from 2000 to 2019 in the Scientific Registry of Transplant Recipients database, the overall BMI reported a "J-shaped" risk profile for graft loss. However, in terms of graft loss and mortality, BMI has been reported to interact with various factors (recipient age, race/ethnicity, sex, and primary renal disease) of recipients. The authors emphasized the importance of personalized risk stratification rather than predicting recipient risk based on absolute BMI alone [26]. Accordingly, our study focused on the presensitization status among recipient factors, and the results showed a significant interaction between recipient BMI and presensitization status, which was an important finding.

In the comparison of baseline characteristics, the D/R BSA ratio was significantly lower in the high BMI-non-sensitized group in this study. Previously, a study had reported that 
if the D/R BSA ratio is less than 0.8 , the allograft outcome is adversely affected [27]. This donor and recipient BSA mismatch may have influenced the allograft outcome in this study, but a high BMI with presensitization status was found to be an independent risk factor when this variable was adjusted in multivariable regression analysis. The rates of DM and HTN as comorbidities were higher in the high BMI groups, and laboratory parameters, such as triglyceride, HDL-cholesterol, and hemoglobin Alc, also showed suitable differences in metabolic syndromes as expected [28]. As reported previously, the rate of previous KT history was higher in the sensitized groups [29]. Lastly, the rate of peritoneal dialysis before transplantation was significantly higher in the high BMI group than in the low BMI group. Weight gain is thought to occur as a consequence of glucose absorption from the peritoneal dialysate in patients undergoing peritoneal dialysis [30].

In the comparison of short-term outcomes, the rates of CMV infection and BK viremia tended to be higher in the HLA presensitized groups. This could be due to immunosuppression caused by desensitization therapy despite adequate prophylaxis in the sensitized groups. In a nationwide cohort study in Korea, desensitization therapy has also been found to be a significant risk factor for infection-related mortality [31]. Early ABMR rates were higher in the sensitized groups, and the rates of late ABMR also tended to be higher in the sensitized groups. These results were consistent with those of previous studies, which showed that the acute rejection rate is higher despite appropriate desensitization therapy in patients with HLA presensitization $[17,31]$. In the comparison of long-term outcomes, a significant decline in allograft function was seen in the high BMI-sensitized group. Additionally, the high BMI-sensitized group had the poorest outcome in adjusted allograft DCGL, and high BMI and presensitization showed significant interaction.

This could be due to several reasons. First, the nephron mass of the donated kidney might be relatively inadequate for recipients with a high BMI. Simply put, a physiologic mismatch between the metabolic demand of the recipient and the nephron mass of the donated kidney may have an adverse allograft outcome [32]. Brenner et al. [33] have previously proposed a nephron underdosing theory for chronic allograft failure. They suggested that the transplantation of kidneys with a relatively large nephron mass compared to the recipients' metabolic demand might lead to tolerance for future immunologic challenges, ischemic events, and CNI toxicities.
Hence, in high BMI recipients with high metabolic demand, kidneys with relatively small nephron mass might be more susceptible to immunologic damage when accompanied by presensitization, resulting in poor allograft outcomes. This is reinforced by the fact that the donated kidney weight/recipient body weight ratio in the high BMI group was significantly low in this study ( $2.6 \pm 0.7$ vs. $3.4 \pm 0.8, \mathrm{p}<0.001$; Supplementary Table 2, available online).

Second, obesity is known to induce alloimmune dysregulation by decreasing adiponectin levels and increasing leptin levels. Adiponectin is known to inhibit B-cell lymphopoiesis, macrophage activation, T-cell proliferative response, and responses of helper T-cell (Th)-1 and Th-2 [34], and serum adiponectin levels are reported to decrease with visceral obesity [35]. In contrast, leptin is known to increase T-cell response, proinflammatory cytokines, recruitment of inflammatory cells, and activities of neutrophils, macrophages, and natural killer cells [34], and is reported to be positively correlated with adipose tissue mass [36]. Previously, a cross-sectional analysis of obese patients and control groups in humans showed a good correlation between leptin level and leukocyte count [37]. Moreover, it has recently been reported that B cells also play an important role in obesity through adipose tissue infiltration and activation [38]. In this study, the relatively rapid decline of the allograft function in the high BMI groups, especially in the high BMI-sensitized group, is probably due to the systemic chronic inflammation in the high BMI group. The presensitization status may interact with chronic inflammation status caused by high BMI, resulting in a worse allograft outcome.

This study has some limitations. First, this was a single-center, retrospective study with a relatively small sample size of only 682 cases. The number of patients in the high BMI-sensitized group was only 21 and only four patients developed graft loss events. However, repeat analysis based on BMI of $\geq 25.0 \mathrm{~kg} / \mathrm{m}^{2}$ showed results that are consistent with previous studies (odds ratio for overall graft loss 1.85, $\mathrm{p}$ $=0.03$; Supplementary Table 3, available online). Additionally, this study is important because it is the first to analyze the relationship between recipient BMI and presensitization status. The second limitation of the study was that it focused on the condition of the recipients before KT and did not analyze whether the patients developed obesity after KT. After KT, prednisolone and other immunosuppressive drugs are administered, which can lead to weight gain. It is not possible to exclude allograft outcomes that are affected by obesity 
developed after KT.

In conclusion, high BMI and HLA sensitization before KT significantly affect long-term allograft outcomes in terms of the decline in allograft function and survival in KT recipients. Our results suggest that active reduction and careful monitoring of BMI might be necessary, especially in patients with high immunologic risk. In the future, studies with larger sample sizes are needed to further clarify the relationship between high BMI and presensitization status.

\section{Conflicts of interest}

All authors have no conflicts of interest to declare.

\section{Funding}

This study was supported by a grant from the Korean Health Technology R\&D Project, Ministry of Health \& Welfare, Republic of Korea (HI20C0317). This manuscript is a research-led paper with no specific participation by funders.

\section{Authors' contributions}

Conceptualization: YP, BHC

Data curation: HL, EJK, SL, THB, JWM, HEY, EJO

Formal analysis: YP, BHC

Funding acquisition: BHC

Investigation: HL, EJK, SL, THB, JWM, HEY, EJO, CWY

Writing-original draft: YP, BHC

Writing-review \& editing: All authors

All authors read and approved the final manuscript.

\section{ORCID}

Yohan Park, https://orcid.org/0000-0001-7416-1841

Hanbi Lee, https://orcid.org/0000-0001-7326-0602

Eun Jeong Ko, https://orcid.org/0000-0002-5604-1296

Sua Lee, https://orcid.org/0000-0001-8983-1457

Tae Hyun Ban, https://orcid.org/0000-0002-2884-4948

Ji-Won Min, https://orcid.org/0000-0001-6295-8095

Hye-Eun Yoon, https://orcid.org/0000-0002-6347-7282

Eun-Jee Oh, https://orcid.org/0000-0001-5870-915X

Chul Woo Yang, https://orcid.org/0000-0001-9796-636X

Byung Ha Chung, https://orcid.org/0000-0003-0048-5717

\section{References}

1. Ng M, Fleming T, Robinson M, et al. Global, regional, and national prevalence of overweight and obesity in children and adults during 1980-2013: a systematic analysis for the Global Burden of Disease Study 2013. Lancet 2014;384:766-781.

2. Korea Disease Control and Prevention Agency (KDCA). Obesity prevalence in Korea (2001-2018), updated January 22, 2020 [Internet]. Cheongju (Korea): KDCA; c2020 [cited 2020 April 2]. Available from: http://www.index.go.kr/potal/main/EachDtlPageDetail.do?idx_cd=2705.

3. Park EK, Yun YS, Kwon SH, et al. Clinicopathologic findings in obesity-related glomerulopathy. Korean J Nephrol 2008;27:4654.

4. Kambham N, Markowitz GS, Valeri AM, Lin J, D'Agati VD. Obesity-related glomerulopathy: an emerging epidemic. Kidney Int 2001;59:1498-1509.

5. Yun HR, Kim H, Park JT, et al. Obesity, metabolic abnormality, and progression of CKD. Am J Kidney Dis 2018;72:400-410.

6. Curran SP, Famure O, Li Y, Kim SJ. Increased recipient body mass index is associated with acute rejection and other adverse outcomes after kidney transplantation. Transplantation 2014;97:64-70.

7. Gore JL, Pham PT, Danovitch GM, et al. Obesity and outcome following renal transplantation. Am J Transplant 2006;6:357363.

8. Hill CJ, Courtney AE, Cardwell CR, et al. Recipient obesity and outcomes after kidney transplantation: a systematic review and meta-analysis. Nephrol Dial Transplant 2015;30:1403-1411.

9. Redondo-Pachón D, Pérez-Sáez MJ, Mir M, et al. Impact of persistent and cleared preformed HLA DSA on kidney transplant outcomes. Hum Immunol 2018;79:424-431.

10. Gloor JM, Cosio FG, Rea DJ, et al. Histologic findings one year after positive crossmatch or $\mathrm{ABO}$ blood group incompatible living donor kidney transplantation. Am J Transplant 2006;6:1841-1847.

11. Ishida H, Furusawa M, Shimizu T, Nozaki T, Tanabe K. Influence of preoperative anti-HLA antibodies on short- and long-term graft survival in recipients with or without rituximab treatment. Transpl Int 2014;27:371-382.

12. Hirai T, Kohei N, Omoto K, Ishida H, Tanabe K. Significance of low-level DSA detected by solid-phase assay in association with acute and chronic antibody-mediated rejection. Transpl Int 2012;25:925-934.

13. Lefaucheur C, Loupy A, Hill GS, et al. Preexisting donor-specific HLA antibodies predict outcome in kidney transplantation. $J$ Am Soc Nephrol 2010;21:1398-1406. 
14. Lee H, Min JW, Kim JI, et al. Clinical significance of HLA-DQ antibodies in the development of chronic antibody-mediated rejection and allograft failure in kidney transplant recipients. Medicine (Baltimore) 2016;95:e3094.

15. Min JW, Lee H, Choi BS, et al. Clinical impact of pre-transplant antibodies against angiotensin II type I receptor and major histocompatibility complex class I-related chain a in kidney transplant patients. Ann Lab Med 2018;38:450-457.

16. Yu JH, Chung BH, Yang CW; Korean Organ Transplantation Registry Study Group. Impact of ABO incompatible kidney transplantation on living donor transplantation. PLoS One 2017;12:e0173878.

17. Chung BH, Choi BS, Oh EJ, et al. Clinical impact of the baseline donor-specific anti-human leukocyte antigen antibody measured by Luminex single antigen assay in living donor kidney transplant recipients after desensitization therapy. Transpl Int 2014;27:49-59.

18. Chung BH, Yun JT, Ha SE, et al. Combined use of rituximab and plasmapheresis pre-transplant increases post-transplant infections in renal transplant recipients with basiliximab induction therapy. Transpl Infect Dis 2013;15:559-568.

19. Sis B, Mengel M, Haas M, et al. Banff '09 meeting report: antibody mediated graft deterioration and implementation of Banff working groups. Am J Transplant 2010;10:464-471.

20. Levey AS, Stevens LA, Schmid CH, et al. A new equation to estimate glomerular filtration rate. Ann Intern Med 2009;150:604-612.

21. Meier-Kriesche HU, Arndorfer JA, Kaplan B. The impact of body mass index on renal transplant outcomes: a significant independent risk factor for graft failure and patient death. Transplantation 2002;73:70-74.

22. Flabouris K, Chadban S, Ladhani M, Cervelli M, Clayton P. Body mass index, weight-adjusted immunosuppression and the risk of acute rejection and infection after kidney transplantation: a cohort study. Nephrol Dial Transplant 2019;34:2132-2143.

23. Nicoletto BB, Fonseca NK, Manfro RC, Gonçalves LF, Leitão CB, Souza GC. Effects of obesity on kidney transplantation outcomes: a systematic review and meta-analysis. Transplantation 2014; 98:167-176.

24. Kim SM, Choi JH, Son MJ, Rim H, Shin HS. Is body mass index a significant independent risk factor for graft failure and patient death in the modern immunosuppressive era? Transplant Proc 2020;52:3058-3068.

25. World Health Organization. Regional Office for the Western Pacific. The Asia-Pacific perspective: redefining obesity and its treatment [Internet]. Sydney: Health Communications Australia; 2000 [cited Sep 9 2020]. Available from: https://apps.who. int/iris/handle/10665/206936.

26. Schold JD, Augustine JJ, Huml AM, et al. Effects of body mass index on kidney transplant outcomes are significantly modified by patient characteristics. Am J Transplant 2021;21:751-765.

27. Jinfeng L, Jia L, Tao G, et al. Donor kidney glomerular filtration rate and donor/recipient body surface area ratio influence graft function in living related kidney transplantation. Ren Fail 2015;37:576-581.

28. Grundy SM. Metabolic syndrome update. Trends Cardiovasc Med 2016;26:364-373.

29. Jordan SC, Pescovitz MD. Presensitization: the problem and its management. Clin J Am Soc Nephrol 2006;1:421-432.

30. Krediet RT, Balafa O. Cardiovascular risk in the peritoneal dialysis patient. Nat Rev Nephrol 2010;6:451-460.

31. Ko EJ, Yu JH, Yang CW, Chung BH; Korean Organ Transplantation Registry Study Group. Clinical outcomes of ABO- and HLA-incompatible kidney transplantation: a nationwide cohort study. Transpl Int 2017;30:1215-1225.

32. Issa N, Vaughan LE, Denic A, et al. Larger nephron size, low nephron number, and nephrosclerosis on biopsy as predictors of kidney function after donating a kidney. Am J Transplant 2019;19:1989-1998.

33. Brenner BM, Milford EL. Nephron underdosing: a programmed cause of chronic renal allograft failure. Am J Kidney Dis 1993;21(5 Suppl 2):66-72.

34. Heinbokel T, Floerchinger B, Schmiderer A, et al. Obesity and its impact on transplantation and alloimmunity. Transplantation 2013;96:10-16.

35. Arita Y, Kihara S, Ouchi N, et al. Paradoxical decrease of an adipose-specific protein, adiponectin, in obesity. Biochem Biophys Res Commun 1999;257:79-83.

36. La Cava A, Matarese G. The weight of leptin in immunity. Nat Rev Immunol 2004;4:371-379.

37. Marzullo P, Minocci A, Giarda P, etal. Lymphocytes and immunoglobulin patterns across the threshold of severe obesity. Endocrine 2014;45:392400.

38. Winer DA, Winer S, Chng MH, Shen L, Engleman EG. B Lymphocytes in obesity-related adipose tissue inflammation and insulin resistance. Cell MolLife Sci 2014;71:1033-1043. 